Quantum Information Processing manuscript No.

(will be inserted by the editor)

\title{
Comment on "Groverian Entanglement Measure and Evolution of Entanglement in Search Algorithm for $n(=3,5)$-Qubit Systems with Real Coefficients" (Volume 6, Number 4, August 2007), by Arti Chamoli and C. M. Bhandari
}

\author{
Preeti Parashar - Swapan Rana \\ Received: / Accepted:
}

\begin{abstract}
We point out that the main results - the analytic expressions for the Groverian Measure of Entanglement, in the above mentioned paper are erroneous. The technical mistake of the paper is discussed. It is shown by an explicit example that the formula for calculating the Groverian measure yields $G(|\psi\rangle)=0$ for some entangled states.
\end{abstract}

\section{What is this communication about?}

In their paper "Groverian Entanglement Measure and Evolution of Entanglement in Search Algorithm for n(=3,5)-Qubit Systems with Real Coefficients" 1], Arti Chamoli and C. M. Bhandari have given an explicit formula for calculating the "Groverian Measure' of entaglement $G(|\psi\rangle)$ in terms of the state coefficients. The derivation of the results involves some maximization process which is very difficult to solve analytically and we will show that unfortunately the authors have missed some crucial technical points which has led to incorrect results.

1.1 The result gives $G(|\psi\rangle)=0$ for some entangled states.

Let us consider the 3-qubit generalized Greenberger-Horne-Zeilinger ( $G G H Z$ ) state given by $\left|\psi_{G G H Z}\right\rangle=a_{000}|000\rangle+a_{111}|111\rangle, a_{000}^{2}+a_{111}^{2}=1$. Then using the formula provided in the paper, we get $P_{\max }\left(\left|\psi_{G G H Z}\right\rangle\right)=1 \Rightarrow G\left(\left|\psi_{G G H Z}\right\rangle\right)=0$, which contradicts the fact that $\left|\psi_{G G H Z}\right\rangle$ is an (genuinely) entangled state. Indeed it is well known 2] that

$$
P_{\max }\left(\left|\psi_{G G H Z}\right\rangle\right)=\max \left\{a_{000}^{2}, a_{111}^{2}\right\} .
$$

Similarly, considering the 5-qubit $G G H Z$ state, the result leads to a contradiction.

Physics and Applied Mathematics Unit

Indian Statistical Institute

203 B T Road

Kolkata 700108

India

E-mail: parashar@isical.ac.in, swapan_r@isical.ac.in 


\section{Where has the error occurred?}

The error has occurred at the maximization stage. The maximization should be with respect to the three independent parameters $\theta_{i}, i=1(1) 3$. If one transforms the three parameters to four parameters, the four parameters cannot be independent and maximizing the function according to the four parameters disregards the functional relations between the parameters. Since $\theta_{w}, \theta_{x}, \theta_{y}, \theta_{z}$ are not independent, the maximum of $P\left(\theta_{1}, \theta_{2}, \theta_{3}\right)$ with respect to $\theta_{1}, \theta_{2}, \theta_{3}$ is in general not equal to the maximum of $P\left(\theta_{w}, \theta_{x}, \theta_{y}, \theta_{z}\right)$ with respect to $\theta_{w}, \theta_{x}, \theta_{y}, \theta_{z}$. The only case when the two maximums agree is the one when the point of maxima $\left(\theta_{w}, \theta_{x}, \theta_{y}, \theta_{z}\right)$ of the four dimensional space lies on a three-dimensional hyperplane. Since the volume of this hyper-plane is zero, the probability of randomly choosing parameters where the optimization with respect to $\theta_{w}, \theta_{x}, \theta_{y}, \theta_{z}$ would give correct result is also zero. Therefore, in the general case, there exists no $\theta_{1}, \theta_{2}, \theta_{3}$ giving $P_{\max }\left(\theta_{w}, \theta_{x}, \theta_{y}, \theta_{z}\right)$.

We will now give an explicit example to elaborate the above discussion. For calculational simplicity, we will consider the $G H Z$ state.

2.1 Explicit calculation of $P_{\max }$ for $G H Z$ state

For the state $\left|\psi_{G H Z}\right\rangle=\frac{1}{\sqrt{2}}(|000\rangle+|111\rangle)$,

$$
P_{\max }\left(\theta_{1}, \theta_{2}, \theta_{3}\right)=\max _{\theta_{1}, \theta_{2}, \theta_{3}} \frac{1}{2}\left[\cos \theta_{1} \cos \theta_{2} \cos \theta_{3}+\sin \theta_{1} \sin \theta_{2} \sin \theta_{3}\right]^{2}
$$

[Note that, from equation (2) it follows by inspection that $P_{\max }=\frac{1}{2}$ and it occurs at $\theta_{i}=0$ or $\left.\theta_{i}= \pm \frac{\pi}{2} \quad \forall i=1,2,3\right]$.

To get the maximum, just following the mentioned paper [1, we get by converting the trigonometric products into sums,

$$
\begin{aligned}
P_{\max }\left(\theta_{1}, \theta_{2}, \theta_{3}\right) & =\max _{\theta_{1}, \theta_{2}, \theta_{3}} \frac{1}{2}\left[\cos \theta_{1} \cos \theta_{2} \cos \theta_{3}+\sin \theta_{1} \sin \theta_{2} \sin \theta_{3}\right]^{2} \\
& =\frac{1}{32}\left[\left(\cos \theta_{w}-\sin \theta_{w}\right)+\left(\cos \theta_{x}+\sin \theta_{x}\right)+\left(\cos \theta_{y}+\sin \theta_{y}\right)\right. \\
& \left.+\left(\cos \theta_{z}-\sin \theta_{z}\right)\right]^{2}
\end{aligned}
$$

where

$$
\begin{aligned}
\theta_{w} & =\theta_{1}+\theta_{2}+\theta_{3} \\
\theta_{x} & =\theta_{1}+\theta_{2}-\theta_{3} \\
\theta_{y} & =\theta_{1}-\theta_{2}+\theta_{3} \\
\theta_{z} & =\theta_{1}-\theta_{2}-\theta_{3}
\end{aligned}
$$

We emphasize that the maximum in (3) should be taken with respect to $\theta_{1}, \theta_{2}, \theta_{3}$ (and not with respect to $\theta_{w}, \theta_{x}, \theta_{y}, \theta_{z}$ ). This is obtained by satisfying $\frac{\partial P}{\partial \theta_{i}}=0$, imposing the constraint

$$
\begin{aligned}
& J_{0}+J_{1}+J_{2}+J_{3}=0 \\
& J_{0}+J_{1}-J_{2}-J_{3}=0 \\
& J_{0}-J_{1}+J_{2}-J_{3}=0
\end{aligned}
$$


or equivalently

$$
J_{0}=-J_{1}=-J_{2}=J_{3}
$$

where

$$
\begin{aligned}
J_{0}=-\sin \theta_{w}-\cos \theta_{w} & =-\sqrt{2} \cos \left(\pi / 4-\theta_{w}\right) \\
J_{1}=-\sin \theta_{x}+\cos \theta_{x} & =\sqrt{2} \cos \left(\pi / 4+\theta_{x}\right) \\
J_{2}=-\sin \theta_{y}+\cos \theta_{y} & =\sqrt{2} \cos \left(\pi / 4+\theta_{y}\right) \\
J_{3}=-\sin \theta_{z}-\cos \theta_{z} & =-\sqrt{2} \cos \left(\pi / 4-\theta_{z}\right)
\end{aligned}
$$

However, the maximum in (3) with respect to $\theta_{w}, \theta_{x}, \theta_{y}, \theta_{z}$ [which has been done by the authors of [1] is obtained by satisfying $\frac{\partial P}{\partial \theta_{w}}=0, \frac{\partial P}{\partial \theta_{x}}=0, \frac{\partial P}{\partial \theta_{y}}=0, \frac{\partial P}{\partial \theta_{z}}=0$ imposing the constraint

$$
J_{0}=J_{1}=J_{2}=J_{3}=0
$$

Clearly the constraints (4) and (5) are not the same. Indeed, there exists no $\theta_{i}, i=1,2,3$ in the range $-\frac{\pi}{2} \leq \theta_{i} \leq \frac{\pi}{2}$ which will satisfy the constraint (5).

[Nevertheless, if we use the erroneous constraint (5) for the maximum with respect to $\theta_{w}, \theta_{x}, \theta_{y}, \theta_{z}$, we will get $P_{\max }=1$, a contradiction that $P_{\max }$ is the square of the maximum possible overlap with a fully separable state].

\subsection{Another paper with similar flaw}

We would like to mention that another paper 3] by the same authors suffers from a similar flaw. In that paper, the authors have derived an analytic formula to calculate $G(|\psi\rangle)$ for arbitrary 4-qubit pure state $|\psi\rangle$ with real coefficients. But due to the similar error (as discussed in first para of Section 2 in the present Comment) in the maximiza-

tion process, the formula (in [3]) is incorrect too. Here we will give just an example to show the discrepancy:

It is well known 2, 4] that for the 4-qubit $W$-state, $G(|W\rangle)=\left(\frac{3}{4}\right)^{3}$. But the formula in [3] gives $G(|W\rangle)=\left(\frac{3}{4}\right)^{2}$.

Thus the formulae presented in the papers [1,3] give incorrect results for both even and odd-qubit cases - as expected.

\section{Conclusion}

Like all other known multi-partite entanglement measures, there is no explicit expression of Groverian measure in terms of the state parameters. We need to go through some maximization process which cannot be achieved analytically for arbitrary parameters. Once the parameters are specified, numerical techniques can be used for maximization. However, for some symmetric states (e.g. GGHZ, Balanced and $W$ states [2], Dicke states [4] the Groverian measure can be calculated analytically. 


\section{References}

1. A. Chamoli and C. M. Bhandari, Quantum Information Processing, Vol. 6, No. 4 (2007).

2. Y. Shimoni, D. Shapira and O. Biham, Phys. Rev. A 69, 062303 (2004).

3. A. Chamoli and C. M. Bhandari, Phys. Lett. A. 346, 17 (2005).

4. P. Parashar and S. Rana, "Groverian measure of entanglement for Dicke states", submitted for publication. 